\title{
Analisys of Hamiltonian Boundary Value Methods (HBVMs): a class of energy-preserving Runge-Kutta methods for the numerical solution of polynomial Hamiltonian systems
}

\author{
L. Brugnano - F. Iavernaro - D. Trigiante
}

Received: September 4, 2009 / Revised: December 2, 2009

\begin{abstract}
One main issue, when numerically integrating autonomous Hamiltonian systems, is the long-term conservation of some of its invariants, among which the Hamiltonian function itself. For example, it is well known that classical symplectic methods can only exactly preserve, at most, quadratic Hamiltonians. In this paper, a new family of methods, called Hamiltonian Boundary Value Methods (HBVMs), is introduced and analyzed. HBVMs are able to exactly preserve, in the discrete solution, Hamiltonian functions of polynomial type of arbitrarily high degree. These methods turn out to be symmetric, precisely $A$-stable, and can have arbitrarily high order. A few numerical tests confirm the theoretical results.
\end{abstract}

Keywords polynomial Hamiltonian · energy drift · symmetric methods · collocation methods · Hamiltonian Boundary Value Methods · energy-preserving methods · one-step methods $\cdot$ Runge-Kutta methods

Mathematics Subject Classification (2000) 65P10 - 65L05

1 Introduction

Omne ignotum pro magnifico

Tacitus, Agr. 30

(sed interdum exitus mathematicae investigationis vere magnifici sunt)

Work developed within the project "Numerical methods and software for differential equations".

Luigi Brugnano

Dipartimento di Matematica “U. Dini”, Università di Firenze, Viale Morgagni 67/A, 50134 Firenze (Italy).

E-mail: luigi.brugnano@unifi.it

Felice Iavernaro

Dipartimento di Matematica, Università di Bari, Via Orabona 4, 70125 Bari (Italy).

E-mail: felix@dm.uniba.it

Donato Trigiante

Dipartimento di Energetica "S. Stecco", Università di Firenze, Via Lombroso 6/17, 50134 Firenze (Italy).

E-mail: trigiant@unifi.it 
The numerical solution of Hamiltonian problems is a relevant issue of investigation since many years: we refer to the recent monographs [8,14] for a comprehensive description of this topic, and to the references therein.

In a certain sense, the use of a numerical method acts as introducing a small perturbation in the original system which, in general, destroys all of its first integrals. The study of the preservation of invariant tori in the phase space of nearly integrable Hamiltonian systems has been a central theme in the research since the pioneering work of Poincaré, the final goal being to asses the stability of the solar system. From a numerical point of view, results in this respect are still poor, and this is justified by considering the delicacy of the problem: as testified by KAM theory, even small Hamiltonian perturbations of completely integrable systems, do not prevent the disappearance of most of the tori, unless a Diophantine condition on the frequencies of the unperturbed system is satisfied.

At the times when research on this topic was started, there were no available numerical methods possessing such conservation features. A main approach to the problem was the devising of symplectic methods. However, though the numerical solution generated by symplectic (and/or symmetric) methods shows some interesting long-time behavior (see, for example, [8, Theorems X.3.1 and XI.3.1]), it was observed that symplecticity alone can only assure, at most, the conservation of quadratic Hamiltonian functions, unless they are coupled with some projection procedure. In the general case, conservation cannot be assured, even though a quasi-preservation can be expected for reversible problems, when symmetric methods are used (see, e.g., [4]). On the other hand, a numerical "drift" can be sometimes observed in the discrete solution [7]. One of the first successful attempts to solve the problem of loss of conservation of the Hamiltonian function by the numerical solution, is represented by discrete gradient methods (see [15] and references therein). Purely algebraic approaches have been also introduced (see, e.g., [6]), without presenting any energypreserving method.

A further approach was considered in [16], where the averaged vector field method was proposed and shown to conserve the energy function of canonical Hamiltonian systems. As was recently outlined (see [5]), approximating the integral appearing in such method by means of a quadrature formula (based upon polynomial interpolation) yields a family of second order Runge-Kutta methods. These latter methods represent an instance of energy-preserving Runge-Kutta methods for polynomial Hamiltonian problems: their first appearance may be found in [10], under the name of $s$-stage trapezoidal methods. Additional examples of fourth and sixth-order RungeKutta methods were presented in [11] and [13].

In [10,11,13], the derivation of such energy preserving Runge-Kutta formulae relies on the definition of the so called "discrete line integral", first introduced in [12]. However, a comprehensive analysis of such methods has not been carried out so far, so that their properties were not known and, moreover, their practical construction was difficult.

In this paper we provide such an analysis, which allows us to derive symmetric methods, of arbitrarily high order, able to preserve Hamiltonian functions of polynomial type, of any specified degree. Such methods are here named Hamiltonian Boundary Value Methods (HBVMs), since the above approach has been, at first, stud- 
ied (see, e.g., [11,13]) in the framework of block Boundary Value Methods. The latter are block one-step methods [3]. However, for the sake of clarity, and later reference, the equivalent Runge-Kutta formulation of HBVMs will be here also considered.

In the remaining part of this section, we introduce the background information concerning the approach. Let then

$$
y^{\prime}=J \nabla H(y), \quad y(0)=y_{0} \in \mathbb{R}^{2 m},
$$

be a Hamiltonian problem in canonical form, where, by setting as usual $I_{m}$ the identity matrix of dimension $m$,

$$
J=\left(\begin{array}{c}
I_{m} \\
-I_{m}
\end{array}\right),
$$

and where the Hamiltonian function, $H(y)$, is a polynomial of degree $v$. It is well known that, for any $y^{*} \in \mathbb{R}^{2 m}$,

$$
H\left(y^{*}\right)-H\left(y_{0}\right)=\int_{y_{0} \rightarrow y^{*}} \nabla H(y)^{T} d y=\int_{0}^{1} \sigma^{\prime}(t)^{T} \nabla H(\sigma(t)) d t,
$$

where $\sigma:[0,1] \rightarrow R^{2 m}$ is any smooth function such that

$$
\sigma(0)=y_{0}, \quad \sigma(1)=y^{*} .
$$

In particular, over a trajectory, $y(t)$, of (1.1), one has

$$
\begin{aligned}
H(y(t))-H\left(y_{0}\right) & =\int_{0}^{t} \nabla H(y(\tau))^{T} y^{\prime}(\tau) d \tau \\
& =\int_{0}^{t} \nabla H(y(\tau))^{T} J \nabla H(y(\tau)) d \tau=0,
\end{aligned}
$$

due to the fact that matrix $J$ in $(1.2)$ is skew-symmetric.

Here we consider the case where $\sigma(t)$ is a polynomial of degree $s$ yielding an approximation to the true solution $y(t)$ in the time interval $[0, h]$ which, without loss of generality, is hereafter normalized to $[0,1]$. More specifically, given the $s+1 \mathrm{ab}-$ scissae

$$
0=c_{0}<c_{1}<\cdots<c_{s}=1,
$$

and the approximations $y_{i} \approx y\left(c_{i}\right), \sigma(t)$ is meant to be defined by the interpolation conditions

$$
\sigma\left(c_{i}\right)=y_{i}, \quad i=0, \ldots, s .
$$

Actually, the approximations $\left\{y_{i}\right\}$ will be unknown, until the new methods will be fully derived.

A different, though related concept, is that of collocating polynomial for the problem, at the abscissae (1.4). Such a polynomial is the unique polynomial $u(t)$, of degree $s+1$, satisfying

$$
u\left(c_{0}\right)=y_{0}, \quad \text { and } \quad u^{\prime}\left(c_{i}\right)=J \nabla H\left(u\left(c_{i}\right)\right), \quad i=0, \ldots, s .
$$


It is well known that (1.6) define a Runge-Kutta collocation method. Moreover, the set of abscissae (1.4) defines a corresponding quadrature formula with weights

$$
b_{i}=\int_{0}^{1} \prod_{j=0, j \neq i}^{s} \frac{t-c_{j}}{c_{i}-c_{j}} d t, \quad i=0,1, \ldots, s,
$$

which has degree of precision ranging from $s$ to $2 s-1$, depending on the choice of the abscissae (1.4). In particular, the highest precision degree is obtained by using the Lobatto abscissae, which we shall consider in the sequel.11 The underlying collocation method has, then, order $2 s$.

Remark 1 Choosing a Gauss distribution of the abscissae $\left\{c_{i}\right\}$ raises the degree of precision of the related quadrature formula to $2 s+1$. In such a case, it is interesting to observe that applying (1.3) along the trajectory $u(t)$ and exploiting the collocation conditions 1.6, one gets

$$
\begin{aligned}
H\left(u\left(c_{s}\right)\right)-H\left(y_{0}\right) & =\int_{0}^{1} u^{\prime}(t)^{T} \nabla H(u(t)) d t \\
& =\sum_{i=0}^{s} b_{i} u^{\prime}\left(c_{i}\right)^{T} \nabla H\left(u\left(c_{i}\right)\right)+R_{S}=R_{S},
\end{aligned}
$$

where $R_{S}$ is the error in the approximation of the line integral. Therefore, $H\left(u\left(c_{S}\right)\right)=$ $H\left(y_{0}\right)$ if and only if $R_{s}=0$, which is implied by assuming that the quadrature formula with abscissae $\left\{c_{i}\right\}$ and weights $\left\{b_{i}\right\}$ is exact when applied to the integrand $u^{\prime}(t)^{T} \nabla H(u(t))$. However, since the integrand has degree

$$
s+(v-1)(s+1)=v(s+1)-1,
$$

it follows that the maximum allowed value for $v$ is 2 . Indeed, it is well known that quadratic invariants are preserved by symmetric collocation methods. On the other hand, when $v>2$, in general $R_{s}$ does not vanish, so that $H\left(u\left(c_{s}\right)\right) \neq H\left(y_{0}\right)$.

The above remark gives us a hint about how to approach the problem. Note that in (1.8) demanding that each term of the sum representing the quadrature formula is null (i.e., the conditions (1.6) ), is an excessive requirement to obtain the conservation property, which causes the observed low degree of precision. A weaker assumption, that would leave the result unchanged, is to relax conditions (1.6) so as to devise a method whose induced quadrature formula, evaluated on a suitable line integral that links two successive points of the numerical solution, is exact and, at the same time, makes the corresponding sum vanish, without requiring that each term is zero

If we use $\sigma(t)$ instead of $u(t)$, the integrand function in (1.3) has degree $v s-1$ so that, in order for the quadrature formula to be exact, one would need say, $k+1$ points, where

$$
k=\left\lceil\frac{v s}{2}\right\rceil,
$$

1 Different choices of the abscissae will be the subject of future investigations.

2 More precisely, in the new methods, conditions 1.6 will turn out to be replaced by relations of the form $\sigma^{\prime}\left(c_{i}\right)=\sum_{j} \beta_{i j} J \nabla H\left(\sigma\left(c_{j}\right)\right)$, which resemble a sort of extended collocation condition (see also [13] Section 2]) since $\sigma^{\prime}\left(c_{i}\right)$ brings information from the global behavior of the problem in the time interval $[0, h]$ (see 3.1-3.8 in Section 3and the analogues in Section 4 . 
if the corresponding Lobatto abscissae are used. Of course, in such a case, the vanishing of the quadrature formula is no longer guaranteed by conditions (1.6) and must be obtained by a different approach. For this purpose, let

$$
r=k-s,
$$

be the number of the required additional points, and let

$$
0<\tau_{1}<\cdots<\tau_{r}<1
$$

be $r$ additional abscissae distinct from (1.4). Moreover, let us define the following silent stages [13],

$$
w_{i} \equiv \sigma\left(\tau_{i}\right), \quad i=1, \ldots, r .
$$

Consequently, the polynomial $\sigma(t)$, which interpolates the couples $\left(c_{i}, y_{i}\right), i=$ $0,1, \ldots, s$, also interpolates the couples $\left(\tau_{i}, w_{i}\right), i=1, \ldots, r$. That is, $\sigma(t)$ interpolates at $k+1$ points, even though it has only degree $s$. If we define the abscissae

$$
\left\{t_{0}<t_{1}<\cdots<t_{k}\right\}=\left\{c_{i}\right\} \cup\left\{\tau_{i}\right\},
$$

and dispose them according to a Lobatto distribution in $[0,1]$ in order to get a formula of degree $2 k$, we have that

$$
\int_{0}^{1} \sigma^{\prime}(t)^{T} \nabla H(\sigma(t)) d t=\sum_{i=0}^{k} b_{i} \sigma^{\prime}\left(t_{i}\right)^{T} \nabla H\left(\sigma\left(t_{i}\right)\right),
$$

and, consequently, the conservation condition becomes

$$
\sum_{i=0}^{k} b_{i} \sigma^{\prime}\left(t_{i}\right)^{T} \nabla H\left(\sigma\left(t_{i}\right)\right)=0
$$

where, now,

$$
b_{i}=\int_{0}^{1} \prod_{j=0, j \neq i}^{k} \frac{t-t_{j}}{t_{i}-t_{j}} d t, \quad i=0,1, \ldots, k .
$$

The left-hand side of (1.15) is called "discrete line integral" because, as will be clear in the sequel, the choice of the path $\sigma(t)$ is dictated by the numerical method by which we will solve problem (1.1) (see [13] for details).

With these premises, in Section 4 , we devise such a method, able to fulfill (1.15), after having set some preliminary results in Section 3 Before that, in Section 2 we state a few facts and notations concerning the shifted Legendre polynomials, which is the framework that we shall use to carry out the analysis of the methods. A few numerical tests are then reported in Section 5 and, finally, a few conclusions are given in Section 6 For sake of completeness, some properties of shifted Legendre polynomials are listed in the Appendix. 


\section{Preliminary results and notations}

The shifted Legendre polynomials, in the interval $[0,1]$, constitute a family of polynomials, $\left\{P_{n}\right\}_{n \in \mathbb{N}}$, for which a number of known properties, named P1-P12, are reported in the Appendix. We now set some notations and results, to be used later.

With reference to the abscissae (1.4), let:

$$
\begin{gathered}
\mathbf{p}_{j}=\left(\begin{array}{c}
P_{j}\left(c_{1}\right) \\
\vdots \\
P_{j}\left(c_{s}\right)
\end{array}\right), \quad \hat{\mathbf{p}}_{j}=\left(\begin{array}{c}
P_{j}\left(c_{0}\right) \\
\mathbf{p}_{j}
\end{array}\right), \quad j=0, \ldots, s, \\
\mathscr{P}_{j}=\left(\mathbf{p}_{0} \ldots \mathbf{p}_{j}\right) \in \mathbb{R}^{s \times j+1}, \quad \hat{\mathscr{P}}_{j}=\left(\hat{\mathbf{p}}_{0} \ldots \hat{\mathbf{p}}_{j}\right) \in \mathbb{R}^{s+1 \times j+1}, \\
\mathbf{I}_{j}=\left(\begin{array}{c}
\int_{0}^{c_{1}} P_{j}(x) d x \\
\vdots \\
\int_{0}^{c_{s}} P_{j}(x) d x
\end{array}\right), \quad \hat{\mathbf{I}}_{j}=\left(\begin{array}{c}
\int_{0}^{c_{0}} P_{j}(x) d x \\
\mathbf{I}_{j}
\end{array}\right) \equiv\left(\begin{array}{c}
0 \\
\mathbf{I}_{j}
\end{array}\right), \quad j=0, \ldots, s .
\end{gathered}
$$

Remark 2 Observe that, from P11, one obtains:

$$
\mathbf{I}_{s}=\mathbf{0}
$$

Furthermore, we set:

$$
\begin{gathered}
\mathscr{I}_{j}=\left(\mathbf{I}_{0} \ldots \mathbf{I}_{j}\right) \in \mathbb{R}^{s \times j+1}, \quad \hat{\mathscr{I}}_{j}=\left(\hat{\mathbf{I}}_{0} \ldots \hat{\mathbf{I}}_{j}\right) \in \mathbb{R}^{s+1 \times j+1}, \\
D_{j}=\left(\begin{array}{ccc}
1 & & \\
3 & & \\
& \ddots & \\
& & 2 j-1
\end{array}\right) \in \mathbb{R}^{j \times j}, \quad \Omega=\left(\begin{array}{ccc}
b_{0} & & \\
& \ddots & \\
& & b_{s}
\end{array}\right),
\end{gathered}
$$

and

$$
G_{j}=\left(\begin{array}{rrrr}
1 & -1 & & \\
1 & 0 & \ddots & \\
& 1 & \ddots & -1 \\
& & \ddots & 0 \\
& & & 1
\end{array}\right) \in \mathbb{R}^{j+1 \times j}
$$

By virtue of $\mathbf{P 3}$ and $\mathbf{P 9}$, we deduce that

$$
\hat{\mathscr{P}}_{j-1}^{T} \Omega \hat{\mathscr{P}}_{j}=\left[D_{j}^{-1} \mathbf{0}\right], \quad j=1, \ldots, s,
$$

and

$$
\hat{\mathscr{I}}_{j-1}=\frac{1}{2} \hat{\mathscr{P}}_{j} G_{j} D_{j}^{-1}, \quad \mathscr{I}_{j-1}=\frac{1}{2} \mathscr{P}_{j} G_{j} D_{j}^{-1}, \quad j=1,2, \ldots
$$


Lemma 1 The matrix $\hat{\mathscr{P}}_{s}=\left(\hat{\mathbf{p}}_{0} \ldots \hat{\mathbf{p}}_{s}\right) \in \mathbb{R}^{s+1 \times s+1}$ is nonsingular. Moreover

$$
\hat{\mathscr{I}}_{s}=\left(\begin{array}{cc}
\mathbf{0}^{T} & 0 \\
\mathscr{I}_{s-1} & \mathbf{0}
\end{array}\right)=\hat{\mathscr{P}}_{s} \hat{G}_{s} \equiv \hat{\mathscr{P}}_{s}\left(\begin{array}{rrrrr}
1 & -1 & 0 & \ldots & 0 \\
1 & 0 & \ddots & \ddots & \vdots \\
\ddots & \ddots & -1 & 0 \\
& \ddots & 0 & 0 \\
& & & 0
\end{array}\right) \in \mathbb{R}^{s+1 \times s+1}
$$

with $\mathscr{I}_{s-1} \in \mathbb{R}^{s \times s}$ a nonsingular matrix.

Proof $\hat{\mathscr{P}}_{s}$ is the transpose of the Gramian matrix defined by the linearly independent polynomials $P_{0}(c), \ldots, P_{s}(c)$ at the distinct abscissae $c_{0}, \ldots, c_{s}$ and is, therefore, nonsingular. The structure of $\hat{\mathscr{I}}_{s}$ follows from (2.4). The matrix $\mathscr{I}_{s-1}$ is nonsingular since, from (2.10), $\hat{\mathscr{P}}_{s}$ is nonsingular, and $\operatorname{rank}\left(\hat{G}_{s}\right)=s$.

\section{Matrix form of collocation methods}

In this section we deliberately do not care of the exactness of the discrete line integral, as stated by (1.14), and in fact we choose $k=s$ (and hence $\left.t_{i}=c_{i}, i=0, \ldots, s\right)$. We show that imposing the vanishing of the discrete line integral (condition (1.15) leads to the definition of the classical Lobatto IIIA methods. The reason why we consider this special situation is that the technique that we are going to exploit is easier to be explained, but at the same time is straightforwardly generalizable to the case $k>s$. As a by-product, we will gain more insight about the link between the new methods and the Lobatto IIIA class. For example, we will deduce that Lobatto IIIA methods (and, in general, all collocation methods) may be defined by means of a polynomial $\sigma(t)$ of degree not larger than that of the collocation polynomial $u(t)$ (indeed, in the present case, $\operatorname{deg} \sigma(t)=\operatorname{deg} u(t)-1)$.

To begin with, let us consider the following expansion of $\sigma^{\prime}(c)$ :

$$
\sigma^{\prime}(c)=\sum_{j=0}^{s-1} \gamma_{j} P_{j}(c)
$$

where the (vector) coefficients $\gamma_{j}$ are to be determined. Then, (1.15) becomes

$$
\sum_{j=0}^{s-1} \gamma_{j}^{T} \sum_{i=0}^{s} b_{i} P_{j}\left(c_{i}\right) \nabla H\left(\sigma\left(c_{i}\right)\right)=0
$$

which will clearly hold true, provided that the following set of orthogonality conditions are satisfied:

$$
\gamma_{j}=\eta_{j} \sum_{i=0}^{s} b_{i} P_{j}\left(c_{i}\right) J \nabla H\left(\sigma\left(c_{i}\right)\right), \quad j=0, \ldots, s-1,
$$


where $\left\{\eta_{j}\right\}$ are suitable scaling factors. We now impose that the polynomial

$$
\sigma(c)=y_{0}+\sum_{j=0}^{s-1} \gamma_{j} \int_{0}^{c} P_{j}(x) d x
$$

satisfies $(1.5)$. By setting

$$
\boldsymbol{\gamma}=\left(\begin{array}{c}
\gamma_{0} \\
\vdots \\
\gamma_{s-1}
\end{array}\right), \quad e=\left(\begin{array}{c}
1 \\
\vdots \\
1
\end{array}\right) \in \mathbb{R}^{s}, \quad \mathbf{y}=\left(\begin{array}{c}
y_{1} \\
\vdots \\
y_{s}
\end{array}\right), \quad \hat{\mathbf{y}}=\left(\begin{array}{c}
y_{0} \\
\mathbf{y}
\end{array}\right) \text {, }
$$

one obtains (see (2.3)- 2.9 )

$$
\mathscr{I}_{s-1} \otimes I_{2 m} \boldsymbol{\gamma}=\left(\frac{1}{2} \mathscr{P}_{s} G_{s} D_{s}^{-1}\right) \otimes I_{2 m} \boldsymbol{\gamma}=\mathbf{y}-e \otimes y_{0} .
$$

Consequently,

$$
\boldsymbol{\gamma}=\left[2 D_{s}\left(\mathscr{P}_{S} G_{S}\right)^{-1}\left(-e I_{S}\right)\right] \otimes I_{2 m} \hat{\mathbf{y}} .
$$

On the other hand, the vector form of relations 3.3 reads

$$
\boldsymbol{\gamma}=\left(\Gamma \hat{\mathscr{P}}_{s-1}^{T} \Omega\right) \otimes \boldsymbol{I}_{2 m} \hat{\mathbf{f}},
$$

where $\Gamma=\operatorname{diag}\left(\eta_{1}, \ldots, \eta_{s}\right) \in \mathbb{R}^{s \times s}$ and

$$
\hat{\mathbf{f}}=\left(f_{0} \ldots f_{s}\right)^{T}, \quad f_{i}=J \nabla H\left(\sigma\left(c_{i}\right)\right), \quad i=0, \ldots, s .
$$

Since $\Gamma$ contains free parameters, we set

$$
\Gamma=D_{s} .
$$

Comparing (3.7) and (3.8), we arrive at the following block method, where now $h$ denotes, in general, the used stepsize,

$$
A \otimes I_{2 m} \hat{\mathbf{y}}=h B \otimes I_{2 m} \hat{\mathbf{f}}
$$

with (see (2.9)

$$
A=\left(-e I_{s}\right), \quad B=\left(\frac{1}{2} \mathscr{P}_{s} G_{s} \hat{\mathscr{P}}_{s-1}^{T} \Omega\right) \equiv\left(\mathscr{I}_{s-1} D_{s} \hat{\mathscr{P}}_{s-1}^{T} \Omega\right) .
$$

The following noticeable result holds true.

Theorem 1 Each row of the block method (3.11)-(3.12) defines a LMF of order $s+1$. The last row corresponds to the Lobatto quadrature formula of order $2 s$. 
Proof For the first part of the proof, it suffices to show that the method is exact for polynomials of degree $s+1$. Clearly, it is exact for polynomials of degree 0 , due to the form of the matrix $A$. We shall then prove that $A \hat{\mathscr{I}}_{s}=B \hat{\mathscr{P}}_{s}$, that is (see 2.3 ), (2.5), and (3.12), $\mathscr{I}_{s}=B \hat{\mathscr{P}}_{s}$. By virtue of (3.12), (2.8), and (2.4), we have

$$
B \hat{\mathscr{P}}_{s}=\mathscr{I}_{s-1} D_{s} \hat{\mathscr{P}}_{s-1}^{T} \Omega \hat{\mathscr{P}}_{s}=\mathscr{I}_{s-1} D_{s}\left[D_{s}^{-1} \mathbf{0}\right]=\left[\mathscr{I}_{s-1} \mathbf{I}_{s}\right]=\mathscr{I}_{s}
$$

which completes the first part of the proof. For the second part, one has to show, by setting as usual $e_{i}$ the $i$ th unit vector, that

$$
e_{s}^{T} B=\left(b_{0} \ldots b_{s}\right),
$$

the vector containing the coefficients of the quadrature formula. From 3.12 , exploiting property $\mathbf{P 7}$ (see also 2.7 ), we obtain

$$
\begin{aligned}
& e_{s}^{T} B=\frac{1}{2} e_{s}^{T} \mathscr{P}_{s} G_{s} \hat{\mathscr{P}}_{s-1}^{T} \Omega=\frac{1}{2}(1 \ldots 1) G_{s} \hat{\mathscr{P}}_{s-1}^{T} \Omega \\
& =e_{1}^{T} \hat{\mathscr{P}}_{s-1}^{T} \Omega=\left(\begin{array}{lll}
1 & \ldots & 1
\end{array}\right) \Omega=\left(b_{0} \ldots b_{s}\right) .
\end{aligned}
$$

As an immediate consequence, the following result follows.

Corollary 1 The block method (3.17)-(3.12) collocates at the Lobatto abscissae (1.4) and has global order 2 s.

Proof The proof follows from known results about collocation methods (see, e.g., [8, Theorem II.1.5]).

Remark 3 In conclusion, the method corresponding to the pencil $(A, B)$, as defined by (3.12), is nothing but the Lobatto IIIA method of order $2 s$.

\subsection{Link between $\sigma(c)$ and the collocation polynomial}

An important consequence of Theorem 1 and Corollary 1 is that the Lobatto IIIA method of order $2 s$ may be also defined by means of an underlying polynomial, namely $\sigma(c)$, of degree $s$ instead of $s+1$, as is the collocation polynomial associated with the method 3.11 .

The main aim of the present subsection is to elucidate the relation between these two polynomials. In what follows, we deliberately ignore the result obtained in Theorem 1 and Corollary 1 so as to provide, among other things, an alternative proof of part of the statements they report 3

Let $u(c)$ be the polynomial (1.6) (of degree $s+1$ ) that collocates problem 1.1) at the abscissae (1.4). The expansion of $u^{\prime}(c)$ along the shifted Legendre polynomials basis reads

$$
u^{\prime}(c)=\sum_{j=0}^{s} \zeta_{j} P_{j}(c)
$$

\footnotetext{
3 The approach exploited in the proof of Theorem 1 turns out to be crucial to deduce the new methods presented in the next section.
} 
Consequently, by setting

$$
\left.\hat{\mathbf{g}}=\left(\begin{array}{c}
g_{0} \\
\vdots \\
g_{s}
\end{array}\right), \quad g_{i}=J \nabla H\left(u\left(c_{i}\right)\right), \quad \text { and } \quad \hat{\zeta} \equiv\left(\begin{array}{c}
\zeta \\
\zeta_{s}
\end{array}\right) \equiv\left(\begin{array}{c}
\zeta_{0} \\
\vdots \\
\zeta_{s-1} \\
\zeta_{s}
\end{array}\right)\right)
$$

one obtains that (1.6) may be recast in matrix notation as $\hat{\mathscr{P}}_{s} \otimes \boldsymbol{I}_{2 m} \hat{\boldsymbol{\zeta}}=\hat{\mathbf{g}}$, or

$$
\hat{\boldsymbol{\zeta}}=\hat{\mathscr{P}}_{s}^{-1} \otimes I_{2 m} \hat{\mathbf{g}} .
$$

We get the expression of $u(c)$ by integrating both sides of (3.13) on the interval $[0, c]$ :

$$
u(c)=y_{0}+\sum_{j=0}^{s-1} \zeta_{j} \int_{0}^{c} P_{j}(x) d x+\zeta_{s} \int_{0}^{c} P_{s}(x) d x .
$$

By virtue of property P11, we get

$$
u\left(c_{i}\right)=y_{0}+\sum_{j=0}^{s-1} \zeta_{j} \int_{0}^{c_{i}} P_{j}(x) d x, \quad i=0, \ldots, s .
$$

Setting $z_{i}=u\left(c_{i}\right), i=1, \ldots, s, \mathbf{z}=\left(z_{1}, \ldots, z_{s}\right)^{T}$, and $\hat{\mathbf{z}}=\left(y_{0}, \mathbf{z}^{T}\right)^{T}$, allows us to recast (3.16) in matrix form. This is done by exploiting a similar argument used to get (3.6) starting from (3.4):

$$
\begin{aligned}
A \otimes I_{2 m} \hat{\mathbf{z}} & =\mathbf{z}-e \otimes y_{0}=\mathscr{I}_{s-1} \otimes I_{2 m} \boldsymbol{\zeta}=\left(\frac{1}{2} \mathscr{P}_{s} G_{s} D_{s}^{-1}\right) \otimes I_{2 m} \boldsymbol{\zeta} \\
& =\left(\frac{1}{2} \mathscr{P}_{s} G_{s}\left[\begin{array}{ll}
D_{s}^{-1} & \mathbf{0}
\end{array}\right]\right) \otimes I_{2 m} \hat{\boldsymbol{\zeta}} .
\end{aligned}
$$

Inserting (3.14) into 3.17, and exploiting 2.8, yields

$$
\begin{aligned}
& A \otimes I_{2 m} \hat{\mathbf{z}} \\
& =\frac{1}{2} \mathscr{P}_{s} G_{s}\left[\begin{array}{ll}
D_{s}^{-1} & \mathbf{0}
\end{array}\right] \hat{\mathscr{P}}_{s}^{-1} \otimes I_{2 m} \hat{\mathbf{g}}=\frac{1}{2} \mathscr{P}_{s} G_{s} \hat{\mathscr{P}}_{s-1}^{T} \Omega \otimes I_{2 m} \hat{\mathbf{g}} \\
& =B \otimes I_{2 m} \hat{\mathbf{g}} .
\end{aligned}
$$

Thus, the collocation problem (1.6) defines the very same method arising from the polynomial $\sigma(c)$ (see 3.11)-3.12) with $h=1$. This implies that system 3.11 is a collocation method defined on the Lobatto abscissae $c_{i}, i=0, \ldots, s$, (therefore, a Lobatto IIIA method), and provides an alternative proof of Corollary 1 In particular, we deduce that

$$
u\left(c_{i}\right)=y_{i}=\sigma\left(c_{i}\right), \quad i=0, \ldots, s .
$$

It follows that 3.15 becomes

$$
u(c)=\sigma(c)+\zeta_{s} \int_{0}^{c} P_{s}(x) d x
$$


and, after differentiating,

$$
u^{\prime}(c)=\sigma^{\prime}(c)+\zeta_{s} P_{s}(c)
$$

We can obtain the expression of the unknown $\zeta_{s}$ by imposing a collocation condition at any of the abscissae $c_{i}$. For example, choosing $c=c_{s}=1$, yields

$$
\zeta_{s}=u^{\prime}(1)-\sigma^{\prime}(1)=f\left(y_{s}\right)-\sum_{j=0}^{s-1} \gamma_{j}=f\left(y_{s}\right)-e^{T} \otimes I_{2 m} \gamma
$$

This latter expression can be slightly simplified by considering that:

(i) $f\left(y_{s}\right)=\left(e^{T} 1\right) \hat{\mathscr{P}}_{s}^{-1} \otimes I_{2 m} \hat{\mathbf{f}}$, which comes from the fact that the system $\hat{\mathscr{P}}_{s}^{T} x=$ $\left(\begin{array}{l}e \\ 1\end{array}\right)$ has solution $x=e_{s+1}$ (the nonsingularity of $\hat{\mathscr{P}}_{s}$ being assured by Lemma 1 ;

(ii) from (3.7) and (3.11-3.12), one has

$$
\begin{aligned}
\boldsymbol{\gamma} & =\left(D_{s} \hat{\mathscr{P}}_{s-1}^{T} \Omega\right) \otimes I_{2 m} \hat{\mathbf{f}}=\left(D_{s} \hat{\mathscr{P}}_{s-1}^{T} \Omega \hat{\mathscr{P}}_{s} \hat{\mathscr{P}}_{s}^{-1}\right) \otimes I_{2 m} \hat{\mathbf{f}} \\
& =\left(D_{s}\left(D_{s}^{-1} \mathbf{0}\right) \hat{\mathscr{P}}_{s}^{-1}\right) \otimes I_{2 m} \hat{\mathbf{f}}=\left(I_{s} \mathbf{0}\right) \hat{\mathscr{P}}_{s}^{-1} \otimes I_{2 m} \hat{\mathbf{f}} .
\end{aligned}
$$

Thus, from 3.20 we get

$$
\zeta_{s}=\left[\left(e^{T} 1\right)-\left(e^{T} 0\right)\right] \hat{\mathscr{P}}_{s}^{-1} \otimes I_{2 m} \hat{\mathbf{f}}=e_{s+1}^{T} \hat{\mathscr{P}}_{s}^{-1} \otimes I_{2 m} \hat{\mathbf{f}} .
$$

The remaining collocation conditions, $u^{\prime}\left(c_{i}\right)=J \nabla H\left(u\left(c_{i}\right)\right), i=0, \ldots, s-1$, are clearly satisfied since the collocation polynomial $u(c)$ is uniquely identified by the $s+2$ linearly independent conditions in (1.6). Nonetheless, they can be easily checked after observing that, from (3.18), (ii), and (3.21),

$$
\hat{\zeta}=\left(\begin{array}{l}
\boldsymbol{\gamma} \\
\zeta_{s}
\end{array}\right)=\hat{\mathscr{P}}_{s}^{-1} \otimes I_{2 m} \hat{\mathbf{f}}
$$

Therefore, from 3.13, 3.14, and 3.18), one obtains,

$$
\hat{\mathbf{u}}^{\prime} \equiv\left(\begin{array}{c}
u^{\prime}\left(c_{0}\right) \\
\vdots \\
u^{\prime}\left(c_{s}\right)
\end{array}\right)=\hat{\mathscr{P}}_{s} \otimes \boldsymbol{I}_{2 m} \boldsymbol{\zeta}=\hat{\mathscr{P}}_{s} \hat{\mathscr{P}}_{s}^{-1} \otimes \boldsymbol{I}_{2 m} \hat{\mathbf{f}}=\hat{\mathbf{f}}
$$

That is (see (3.9)), $u^{\prime}\left(c_{i}\right)=J \nabla H\left(u\left(c_{i}\right)\right), i=0, \ldots, s$.

\section{Derivation of the methods}

The arguments for deriving the methods in the general case where $k \geq s$ (which makes the discrete line integral exact) are a straightforward extension of what stated above. In particular, let us consider again the expansion (3.1)-(3.4) of the polynomial $\sigma(c)$. Then, condition (1.15) can be recast as (compare with 3.2)

$$
\sum_{j=0}^{s-1} \gamma_{j}^{T} \sum_{i=0}^{k} b_{i} P_{j}\left(t_{i}\right) \nabla H\left(\sigma\left(t_{i}\right)\right)=0
$$


which will clearly hold true, provided that the following set of orthogonality conditions are satisfied (compare with (3.3), see also (1.16)):

$$
\gamma_{j}=\eta_{j} \sum_{i=0}^{k} b_{i} P_{j}\left(t_{i}\right) J \nabla H\left(\sigma\left(t_{i}\right)\right), \quad j=0, \ldots, s-1,
$$

where $\left\{\eta_{j}\right\}$ are suitable scaling factors. According to (3.10), we choose them as $\eta_{j}=2 j+1, j=0, \ldots, s-1$. The vector $\boldsymbol{\gamma}$ (see (3.5) ) is then obtained by imposing that the polynomial $\sigma(c)$ in (3.4) satisfies the interpolation constrains (1.5) and (1.12). In so doing, one obtains a block method characterized by the pencil $(A, B)$, where the two $k \times k+1$ matrices $A$ and $B$ are defined as follows. In order to simplify the notation, we shall use a "Matlab-like" notation: let ind s $_{s} \in \mathbb{R}^{s+1}$ and ind $d_{r} \in \mathbb{R}^{r}$ be the vectors whose entries are the indexes of the main abscissae $c_{0}<\cdots<c_{s}$ in (1.4) and of the silent ones $\tau_{1}<\cdots<\tau_{r}$ in (1.11), respectively, within the Lobatto abscissae $t_{0}<\cdots<t_{k}$, as defined in (1.13). Then, the orthogonality conditions (4.2) will define the first $s$ rows of $A$ and $B 4$ (compare with 3.12 ):

$$
A\left(1: s, \text { ind }_{s}\right)=\left(-e I_{s}\right), \quad B(1: s,:)=\left(\mathscr{I}_{s-1} D_{s} \overline{\mathscr{P}}^{T} \bar{\Omega}\right),
$$

where (see (2.5)-(2.6) and (1.13)

$$
\overline{\mathscr{P}}=\left(\begin{array}{ccc}
P_{0}\left(t_{0}\right) & \ldots & P_{s-1}\left(t_{0}\right) \\
\vdots & & \vdots \\
P_{0}\left(t_{k}\right) & \ldots & P_{s-1}\left(t_{k}\right)
\end{array}\right) \in \mathbb{R}^{k+1 \times s},
$$

and (see 1.7)

$$
\bar{\Omega}=\left(\begin{array}{ccc}
b_{0} & & \\
& \ddots & \\
& & b_{k}
\end{array}\right) \in \mathbb{R}^{k+1 \times k+1} .
$$

On the other hand, the interpolation conditions for the silent stages 1.12 define the last $r$ rows of the matrix $A$ (the corresponding rows of $B$ are obviously zero):

$$
\begin{aligned}
& A\left(s+1: k, \text { ind }_{r}\right)=I_{r}, \\
& A\left(s+1: k, \text { ind }_{s}\right)=-\overline{\mathscr{I}}_{r}\left[\mathscr{I}_{s-1}^{-1}\left(-e I_{s}\right)\right]-\bar{e} \cdot e_{1}^{T},
\end{aligned}
$$

where $I_{r}$ is the identity matrix of dimension $r, \bar{e}=(1, \ldots, 1)^{T} \in \mathbb{R}^{r}, e_{1}$ is the first unit vector (of dimension $s+1$ ), and

$$
\overline{\mathscr{I}}_{r}=\left(\begin{array}{ccc}
\int_{0}^{\tau_{1}} P_{0}(x) d x & \ldots & \int_{0}^{\tau_{1}} P_{s-1}(x) d x \\
\vdots & & \vdots \\
\int_{0}^{\tau_{r}} P_{0}(x) d x & \ldots & \int_{0}^{\tau_{r}} P_{s-1}(x) d x
\end{array}\right) \in \mathbb{R}^{r \times s} .
$$

The following result generalizes Theorem 1 to the present setting (the proof being similar).

\footnotetext{
4 As a further convention, the entries not explicitly set are assumed to be 0 .
} 
Theorem 2 Each row of the block method (4.3)-(4.6) defines a LMF of order at least $s$. The s-th row corresponds to the Lobatto quadrature formula of order $2 k$.

Definition 1 We call the method defined by the pencil $(A, B)$ in (4.3)-(4.6) $a$ "Hamiltonian BVM with $k$ steps and degree $s$ ", hereafter $\operatorname{HBVM}(k, s) 5$

Remark 4 The structure of the nonlinear system associated with the $\operatorname{HBVM}(k, s)$ is better visualized by performing a permutation of the stages that splits, into two block sub-vectors, the fundamental stages and the silent ones. More precisely, the permuted vector of stages, say $\mathbf{z}$, is required to be:

$$
\mathbf{z}=[\underbrace{y_{0}^{T}, y_{1}^{T}, \ldots, y_{s}^{T}}_{\text {fundamental stages }}, \underbrace{w_{1}^{T}, w_{2}^{T}, \ldots, w_{r}^{T}}_{\text {silent stages }}]^{T} \equiv\left[y_{0}^{T}, \mathbf{y}^{T}, \mathbf{w}^{T}\right]^{T} .
$$

This is accomplished by introducing the permutation matrices $W \in \mathbb{R}^{k \times k}$ and $W_{1} \in$ $\mathbb{R}^{k+1 \times k+1}$, such that

$$
W\left(\begin{array}{c}
2 \\
\vdots \\
k+1
\end{array}\right)=\left(\begin{array}{c}
\operatorname{ind}_{s}(2: s+1) \\
\operatorname{ind}_{r}
\end{array}\right), \quad W_{1}\left(\begin{array}{c}
1 \\
\vdots \\
k+1
\end{array}\right)=\left(\begin{array}{c}
\operatorname{ind}_{s} \\
\operatorname{ind}_{r}
\end{array}\right) .
$$

It is easy to realize that

$$
W A W_{1}^{T}=\left(\begin{array}{ccc}
-e & I_{s} & 0_{s \times r} \\
-a_{0} & -A_{1} & I_{r}
\end{array}\right), \quad W B W_{1}^{T}=\left(\begin{array}{ccc}
b_{0} & B_{1} & B_{2} \\
\mathbf{0} & 0_{r \times s} & 0_{r \times r}
\end{array}\right),
$$

where $\left[-a_{0},-A_{1}\right]$ coincides with $A\left(s+1: k\right.$, ind $\left._{s}\right)$ in (4.6), while $\left[b_{0}, B_{1}, B_{2}\right]$ matches the matrix $B(1: s,:)$ in (4.3). The $\operatorname{HBVM}(k, s)$ then takes the form:

$$
\left(\begin{array}{ccc}
-e & I_{s} & 0_{s \times r} \\
-a_{0} & -A_{1} & I_{r}
\end{array}\right) \otimes I_{2 m} \mathbf{z}=h\left(\begin{array}{ccc}
b_{0} & B_{1} & B_{2} \\
\mathbf{0} & 0_{r \times s} & 0_{r \times r}
\end{array}\right) \otimes J \nabla H(\mathbf{z}) .
$$

The presence of the null blocks in the lower part of $W B W_{1}^{T}$ clearly suggests that the (generally nonlinear) system (4.7) of (block) size $k$ is actually equivalent to a system having (block) size s. Indeed, we can easily remove the silent stages,

$$
\mathbf{w}=a_{0} \otimes y_{0}+A_{1} \otimes I_{2 m} \mathbf{y}
$$

and obtain

$$
\begin{aligned}
\mathbf{y}=e \otimes y_{0}+ & h b_{0} \otimes\left(J \nabla H\left(y_{0}\right)\right)+h B_{1} \otimes J \nabla H(\mathbf{y}) \\
+ & h B_{2} \otimes J \nabla H\left(a_{0} \otimes y_{0}+A_{1} \otimes I_{2 m} \mathbf{y}\right) .
\end{aligned}
$$

We refer to [2] for an alternative technique to reduce the dimension of system 4.7].

\footnotetext{
5 Indeed, the pencil $(A, B)$ perfectly fits the framework of block BVMs (see, e.g., [3]).
} 
Remark 5 As was shown in the previous section, when $k=s$, the $H B V M(s, s)$ coincides with the Lobatto IIIA method of order $2 s$. More in general, for $k \geq s$, by summing up (4.3)-(4.6), we can cast HBVM $(k, s)$ as a Runge-Kutta method with the following tableau:

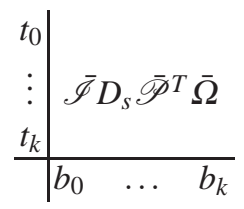

where

$$
\overline{\mathscr{I}}=\left(\begin{array}{ccc}
\int_{0}^{t_{0}} P_{0}(x) d x & \ldots & \int_{0}^{t_{0}} P_{s-1}(x) d x \\
\vdots & & \vdots \\
\int_{0}^{t_{k}} P_{0}(x) d x & \ldots & \int_{0}^{t_{k}} P_{s-1}(x) d x
\end{array}\right) \in \mathbb{R}^{k+1 \times s} .
$$

We observe that the $k+1 \times k+1$ matrix

$$
C=\overline{\mathscr{I}} D_{s} \overline{\mathscr{P}}^{T} \bar{\Omega}
$$

appearing in (4.9) has rank s, thus confirming that the computational cost per iteration depends on $s$, rather than on $k$ (see [2] for more details and a practical example of Butcher tableau concerning the method $H B V M(6,2))$.

By the way, we observe that, when $s=1, \operatorname{HBVM}(k, 1)$ are nothing but the " $s$-stage trapezoidal methods", defined in [10], based on the Lobatto abcissae. In such a case, the matrix $C$ becomes

$$
C=\left(\begin{array}{c}
t_{0} \\
\vdots \\
t_{k}
\end{array}\right)\left(\begin{array}{lll}
b_{0} & \ldots & b_{k}
\end{array}\right) .
$$

Similarly, for $s=2$ and $k=4, \operatorname{HBVM}(4,2)$ coincides with the fourth-order method presented in [13. Section 4.2], able to preserve polynomial Hamiltonians of degree four.

Concerning the order of convergence, the following result generalizes that of Corollary 1

Corollary 2 The $\operatorname{HBVM}(k, s)$ (4.3)-(4.6) has order of convergence $p=2 s$.

Proof By virtue of Theorem 2 , the corresponding Runge-Kutta method (4.9) satisfies the usual simplifying assumptions $B(2 k)$ and $C(s)$. If we are able to prove $D(s-1)$, from the classical result of Buthcher (see, e.g., [9, Theorem 5.1]), it will follow that the method has order $p=2 s$. With reference to (4.9), the condition $D(s-1)$ can be cast in matrix form, by introducing the vectors $e=(1, \ldots, 1)^{T} \in \mathbb{R}^{s-1}, \bar{e}=$ $(1, \ldots, 1)^{T} \in \mathbb{R}^{k+1}$, and the matrices

$$
Q=\operatorname{diag}(1, \ldots, s-1), \quad T=\operatorname{diag}\left(t_{0}, \ldots, t_{k}\right), \quad V=\left(t_{i-1}^{j-1}\right) \in \mathbb{R}^{k+1 \times s-1},
$$

as

$$
Q V^{T} \bar{\Omega}\left(\overline{\mathscr{I}} D_{s} \overline{\mathscr{P}}^{T} \bar{\Omega}\right)=\left(e \bar{e}^{T}-V^{T} T\right) \bar{\Omega},
$$


i.e.,

$$
\overline{\mathscr{P}}_{D_{s}} \overline{\mathscr{I}}^{T} \bar{\Omega} V Q=\left(\bar{e} e^{T}-T V\right) .
$$

Since the quadrature is exact for polynomials of degree $2 s-1 \leq 2 k-1$, one has

$$
\begin{aligned}
\left(\overline{\mathscr{I}}^{T} \bar{\Omega} V Q\right)_{i j} & =\left(\sum_{\ell=0}^{k} b_{\ell} \int_{0}^{t_{\ell}} P_{i-1}(x) \mathrm{d} x\left(j t_{\ell}^{j-1}\right)\right)=\left(\int_{0}^{1} \int_{0}^{t} P_{i-1}(x) \mathrm{d} x\left(j t^{j-1}\right) \mathrm{d} t\right) \\
& =\left(\delta_{i 1}-\int_{0}^{1} P_{i-1}(x) x^{j} \mathrm{~d} x\right), \quad i=1, \ldots, s, \quad j=1, \ldots, s-1,
\end{aligned}
$$

where the last equality is obtained by integrating by parts, with $\delta_{i 1}$ the Kronecker symbol. Consequently,

$$
\begin{array}{r}
\left(\overline{\mathscr{P}} D_{s} \overline{\mathscr{I}}^{T} \bar{\Omega} V Q\right)_{i j}=\left(1-\sum_{\ell=0}^{s-1} \eta_{\ell} P_{\ell}\left(t_{i}\right) \int_{0}^{1} P_{\ell}(x) x^{j} \mathrm{~d} x\right)=\left(1-t_{i-1}^{j}\right), \\
i=1, \ldots, k+1, \quad j=1, \ldots, s-1,
\end{array}
$$

that is, 4.11, where the last equality follows from the fact that

$$
\sum_{\ell=0}^{s-1} \eta_{\ell} P_{\ell}(t) \int_{0}^{1} P_{\ell}(x) x^{j} \mathrm{~d} x=t^{j}, \quad j=1, \ldots, s-1 .
$$

An additional, remarkable property of such methods is gained, provided that the abscissae $\left\{t_{0}, \ldots, t_{k}\right\}$ (1.13) are symmetrically distributed (as is the case of the Lobatto abscissae here considered). For this purpose, we need to introduce some notations and preliminary results. Let us define the matrix

$$
E_{n}=\left(\begin{array}{cc} 
& 1 \\
\cdot & \cdot \\
1 &
\end{array}\right) \in \mathbb{R}^{n \times n}
$$

which, when applied to a vector of length $n$, reverses the order of its entries. We also set

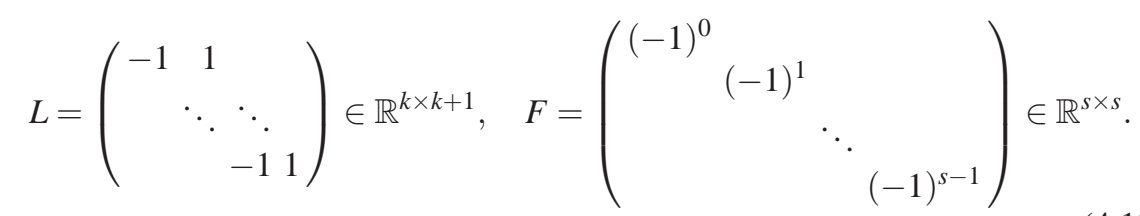

The following preliminary result holds true.

Lemma 2 If the abscissae (1.13) are symmetric, then matrix (4.10) satisfies:

$$
E_{k} L C E_{k+1}=L C \text {. }
$$



(4.5)

Proof From the symmetry of the abscissae it easily follows that (see (1.16) and

$$
E_{k+1} \bar{\Omega} E_{k+1}=\bar{\Omega} .
$$

From property P6, we have that (see 4.4)

$$
\overline{\mathscr{P}}^{T} E_{k+1}=F \overline{\mathscr{P}}^{T} .
$$

Moreover, by considering that (see (1.4))

$$
L \mathscr{I}=\left(\begin{array}{ccc}
\int_{t_{0}}^{t_{1}} P_{0}(x) d x & \ldots & \int_{t_{0}}^{t_{1}} P_{s-1}(x) d x \\
\vdots & & \vdots \\
\int_{t_{k-1}}^{t_{k}} P_{0}(x) d x & \ldots & \int_{t_{k-1}}^{t_{k}} P_{s-1}(x) d x
\end{array}\right),
$$

again from $\mathbf{P 6}$ we see that

$$
E_{S} L \mathscr{I}=L \mathscr{I} F
$$

Finally, from (4.10) we obtain

$$
\begin{aligned}
& E_{k} L C E_{k+1}= \\
& =\left(E_{k} L \mathscr{I}\right) D_{s}\left(\overline{\mathscr{P}}^{T} E_{k+1}\right)\left(E_{k+1} \bar{\Omega} E_{k+1}\right) \\
& =L \mathscr{I} F D_{s} F \overline{\mathscr{P}}^{T} \bar{\Omega}=L \mathscr{I} D_{s} \overline{\mathscr{P}}^{T} \bar{\Omega}=L C .
\end{aligned}
$$

As a consequence, we have the following result.

Theorem 3 If the abscissae (1.13) are symmetric, then the method (4.3)-(4.6) (i.e., (4.9)) is symmetric, that is, it is self-adjoint.

Proof Indeed, the discrete solution, $\hat{\mathbf{y}}$, satisfies the equation (see 4.9) 4.10 and (4.12)

$$
L \otimes I_{2 m} \hat{\mathbf{y}}=h L C \otimes I_{2 m} f(\hat{\mathbf{y}}) .
$$

Considering that $E_{k} L E_{k+1}=-L$ and, from Lemma $2, E_{k} L C E_{k+1}=L C$, one then obtains

$$
\begin{aligned}
L \otimes I_{2 m}\left(E_{k+1} \otimes I_{2 m} \hat{\mathbf{y}}\right) & =-h L C \otimes I_{2 m}\left(E_{k+1} \otimes I_{2 m} f(\hat{\mathbf{y}})\right) \\
& =-h L C \otimes I_{2 m} f\left(E_{k+1} \otimes I_{2 m} \hat{\mathbf{y}}\right) .
\end{aligned}
$$

The thesis then follows by observing that the vector $E_{k+1} \otimes I_{2 m} \hat{\mathbf{y}}$ contains the timereversed discrete solution.

The next theorem summarizes the results about $\operatorname{HBVM}(k, s)$.

Theorem 4 (Main Result) For all $s=1,2, \ldots$, and $k \geq s$, the $H B V M(k, s)$ method:

1. has order of accuracy 2 s;

2. is energy-preserving for polynomial Hamiltonians of degree not larger than $2 k / s$;

3. for general $C^{(2 k+1)}$ Hamiltonians, the energy error at each integration step is $O\left(h^{2 k+1}\right)$, if $h$ is the used stepsize; 6

\footnotetext{
${ }^{6}$ Consequently, on any finite interval the global energy error is not larger than $O\left(h^{2 k}\right)$.
} 
4. is symmetric and, therefore, precisely A-stable.

Proof Item 1 follows from Corollary 2 Item 2 follows from the fact that, for such polynomial Hamiltonians, the vanishing discrete line integral equals the continuous line integral (see (1.14) and (1.15). Similarly, Item 3 follows from the fact that, by using arguments similar to those used in Remark 1 (see (1.8)), the energy error per integration step equals the quadrature error of the Gauss-Lobatto formula of order $2 k$. Finally, Item 4 follows from Theorem 3 , since the Lobatto abscissae $\left\{t_{i}\right\}$ are symmetrically distributed.

Remark 6 From the result of Theorem 4 we can then concude that $\operatorname{HBVM}(k, s)$ is optimal, both from the point of view of the order and stability properties. Moreover, its computational cost, as observed in Remarks 4 and $[5]$ is seen to depend on s, rather than on $k$.

\section{Numerical Tests}

We here report a few numerical tests, in order to show the potentialities of $\operatorname{HBVM}(k, s)$.

Let then consider, at first, the Hamiltonian problem characterized by the polynomial Hamiltonian (4.1) in [7],

$$
H(p, q)=\frac{p^{3}}{3}-\frac{p}{2}+\frac{q^{6}}{30}+\frac{q^{4}}{4}-\frac{q^{3}}{3}+\frac{1}{6},
$$

having degree $v=6$, starting at the initial point $y_{0} \equiv(q(0), p(0))^{T}=(0,1)^{T}$. For such a problem, in [7] it has been experienced a numerical drift in the discrete Hamiltonian, when using the fourth-order Lobatto IIIA method7 with stepsize $h=0.16$. This is confirmed by the plot in Figure 5.1, where a linear drift in the numerical Hamiltonian is clearly observable. On the other hand, by using the fourth-order $\operatorname{HBVM}(6,2)$ with the same stepsize, the drift disappears, as shown in Figure 5.2. since such method exactly preserves polynomial Hamiltonians of degree up to 6 . Moreover, the order of convergence $p=4$ is (numerically) confirmed by the results listed in Table 5.1 where the used stepsizes $h$, the maximum estimated error (obtained as the difference of two consecutive solutions), and the estimated order of convergence are listed.

The second test problem is the Fermi-Pasta-Ulam problem (see [8, Section I.5.1]), defined by the Hamiltonian

$$
H(p, q)=\frac{1}{2} \sum_{i=1}^{m}\left(p_{2 i-1}^{2}+p_{2 i}^{2}\right)+\frac{\omega^{2}}{4} \sum_{i=1}^{m}\left(q_{2 i}-q_{2 i-1}\right)^{2}+\sum_{i=0}^{m}\left(q_{2 i+1}-q_{2 i}\right)^{4},
$$

with $q_{0}=q_{2 m+1}=0, m=3, \omega=50$, and starting vector

$$
p_{i}=0, \quad q_{i}=(i-1) / 10, \quad i=1, \ldots, 6 .
$$

In such a case, the Hamiltonian function is a polynomial of degree 4, so that the fourth-order $\operatorname{HBVM}(4,2)$ method, which is used with stepsize $h=0.05$, is able to

\footnotetext{
7 Such method coincides with the $\operatorname{HBVM}(2,2)$ above described.
} 


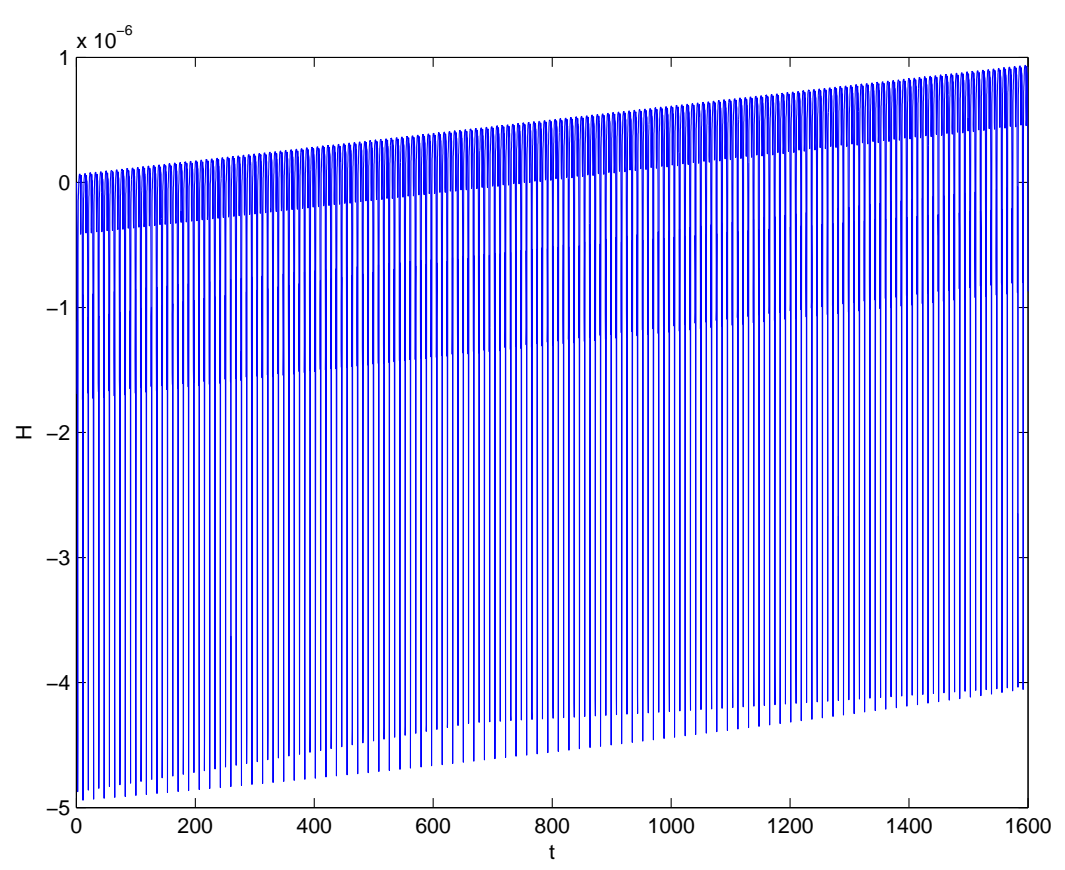

Fig. 5.1 Fourth-order Lobatto IIIA method, $h=0.16$, problem 5.1.

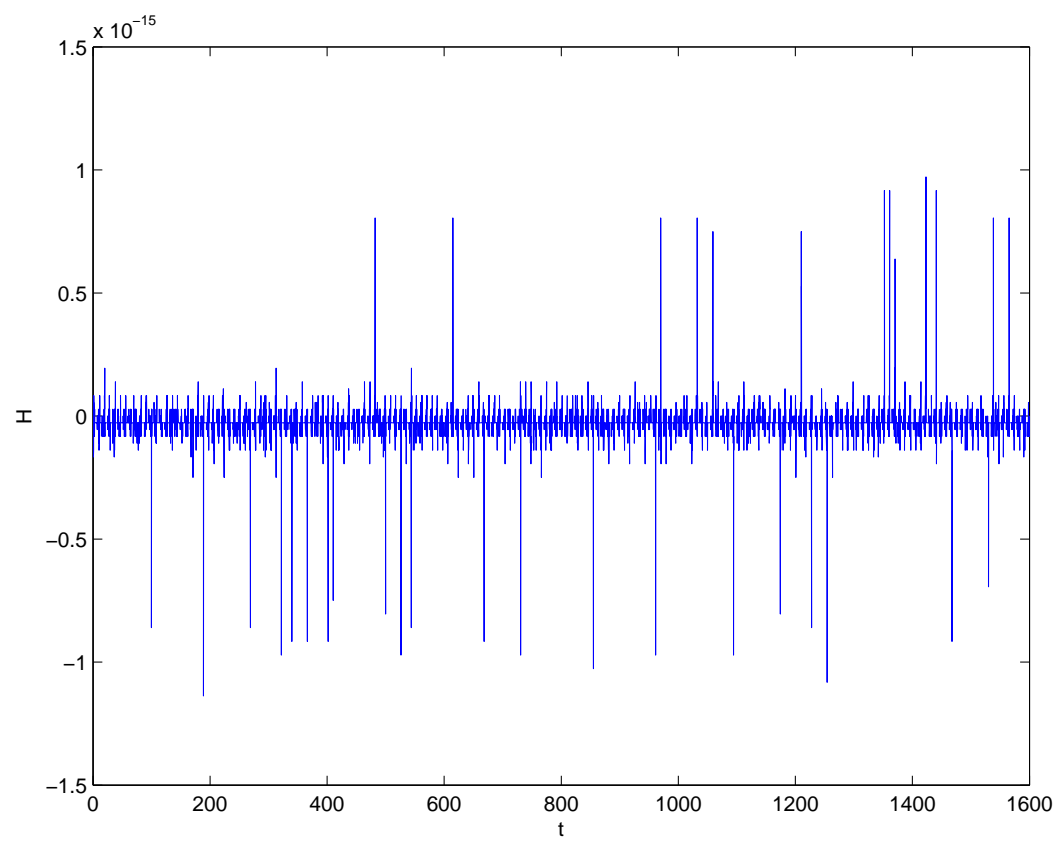

Fig. 5.2 Fourth-order $\operatorname{HBVM}(6,2)$ method, $h=0.16$, problem 5.1. 
Table 5.1 Numerical order of convergence for the $\operatorname{HBVM}(6,2)$ method, problem 5.1.

\begin{tabular}{|c|lllll|}
\hline$h$ & 0.32 & 0.16 & 0.08 & 0.04 & 0.02 \\
\hline error & $2.288 \cdot 10^{-2}$ & $1.487 \cdot 10^{-3}$ & $9.398 \cdot 10^{-5}$ & $5.890 \cdot 10^{-6}$ & $3.684 \cdot 10^{-7}$ \\
\hline order & - & 3.94 & 3.98 & 4.00 & 4.00 \\
\hline
\end{tabular}

Table 5.2 Numerical order of convergence for the $\operatorname{HBVM}(4,2)$ method, problem 5.2.

\begin{tabular}{|c|lllll|}
\hline$h$ & $1.6 \cdot 10^{-2}$ & $8 \cdot 10^{-3}$ & $4 \cdot 10^{-3}$ & $2 \cdot 10^{-3}$ & $10^{-3}$ \\
\hline error & 3.030 & $1.967 \cdot 10^{-1}$ & $1.240 \cdot 10^{-2}$ & $7.761 \cdot 10^{-4}$ & $4.853 \cdot 10^{-5}$ \\
\hline order & - & 3.97 & 3.99 & 4.00 & 4.00 \\
\hline
\end{tabular}

Table 5.3 Numerical order of convergence for the $\operatorname{HBVM}(6,2)$ method, problem 5.3 .

\begin{tabular}{|c|lllll|}
\hline$h$ & $3.2 \cdot 10^{-2}$ & $1.6 \cdot 10^{-2}$ & $8 \cdot 10^{-3}$ & $4 \cdot 10^{-3}$ & $2 \cdot 10^{-3}$ \\
\hline error & $3.944 \cdot 10^{-6}$ & $2.635 \cdot 10^{-7}$ & $1.729 \cdot 10^{-8}$ & $1.094 \cdot 10^{-9}$ & $6.838 \cdot 10^{-11}$ \\
\hline order & - & 3.90 & 3.93 & 3.98 & 4.00 \\
\hline
\end{tabular}

exactly preserve the Hamiltonian, as confirmed by the plot in Figure 5.4 whereas the fourth-order Lobatto IIIA method provides the result plotted in Figure 5.3 Moreover, in Table 5.2 we list corresponding results as in Table 5.1, again confirming the fourthorder convergence.

In the previous examples, the Hamiltonian function was a polynomial. Nevertheless, as is easily argued from Theorem $4 \operatorname{HBVM}(k, s)$ are expected to produce a practical conservation of the energy when applied to systems defined by a non-polynomial Hamiltonian function which are sufficiently differentiable. As an example, we consider the motion of a charged particle in a magnetic field with Biot-Savart potential 8 It is defined by the Hamiltonian

$$
\begin{aligned}
& H(x, y, z, \dot{x}, \dot{y}, \dot{z})= \\
& \frac{1}{2 m}\left[\left(\dot{x}-\alpha \frac{x}{\rho^{2}}\right)^{2}+\left(\dot{y}-\alpha \frac{y}{\rho^{2}}\right)^{2}+(\dot{z}+\alpha \log (\rho))^{2}\right],
\end{aligned}
$$

with $\rho=\sqrt{x^{2}+y^{2}}, \alpha=e B_{0}, m$ is the particle mass, $e$ is its charge, and $B_{0}$ is the magnetic field intensity. We have used the values

$$
m=1, \quad e=-1, \quad B_{0}=1,
$$

with starting point

$$
x=0.5, \quad y=10, \quad z=0, \quad \dot{x}=-0.1, \quad \dot{y}=-0.3, \quad \dot{z}=0 .
$$

In Figure 5.5 the trajectory of the particle in the interval $\left[0,10^{3}\right]$ is plotted in the phase space. As one can see, it is a helix that wings downward. By using the fourthorder Lobatto IIIA method with stepsize $h=0.1$, a drift in the numerical Hamiltonian can be again observed (see Figure 5.6), so that the method does introduce a friction. When using the $\operatorname{HBVM}(4,2)$ method with the same stepsize, the drift disappears and the Hamiltonian turns out to be almost preserved (see Figure 5.7). As expected, the

\footnotetext{
8 As an example, this kind of motion causes the well known phenomenon of aurora borealis.
} 


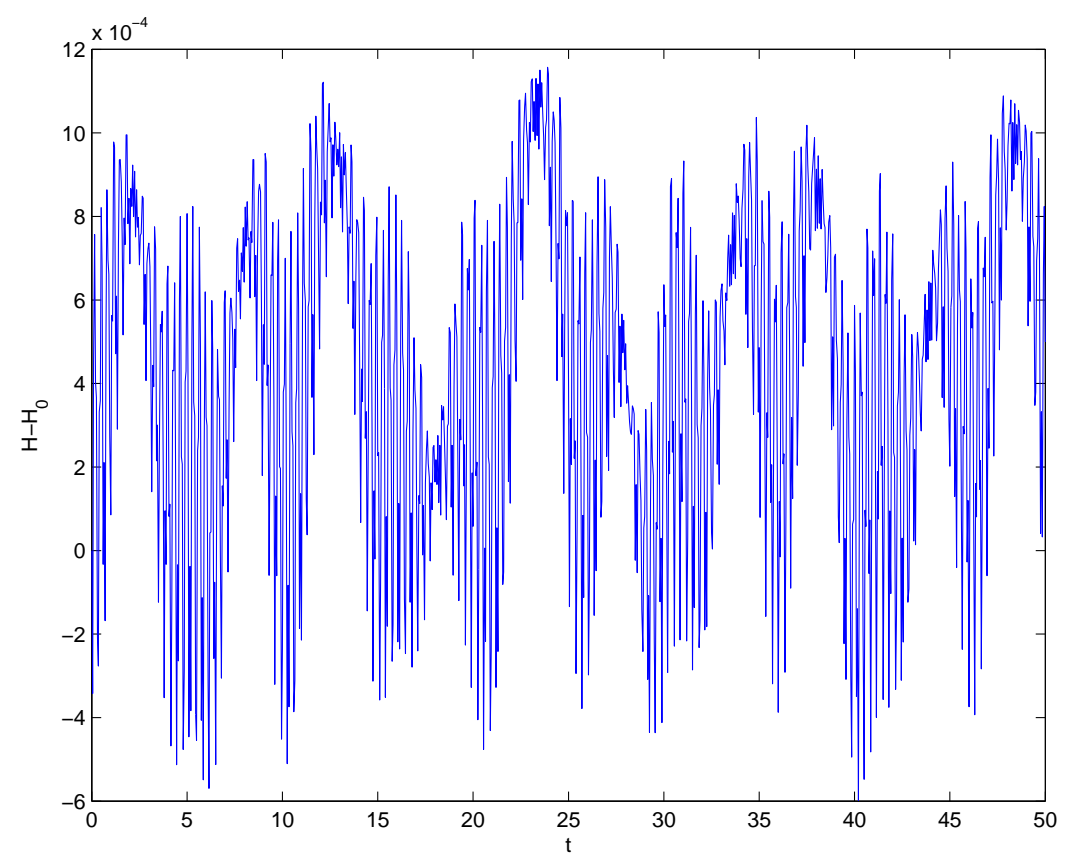

Fig. 5.3 Fourth-order Lobatto IIIA method, $h=0.05$, problem 5.2.

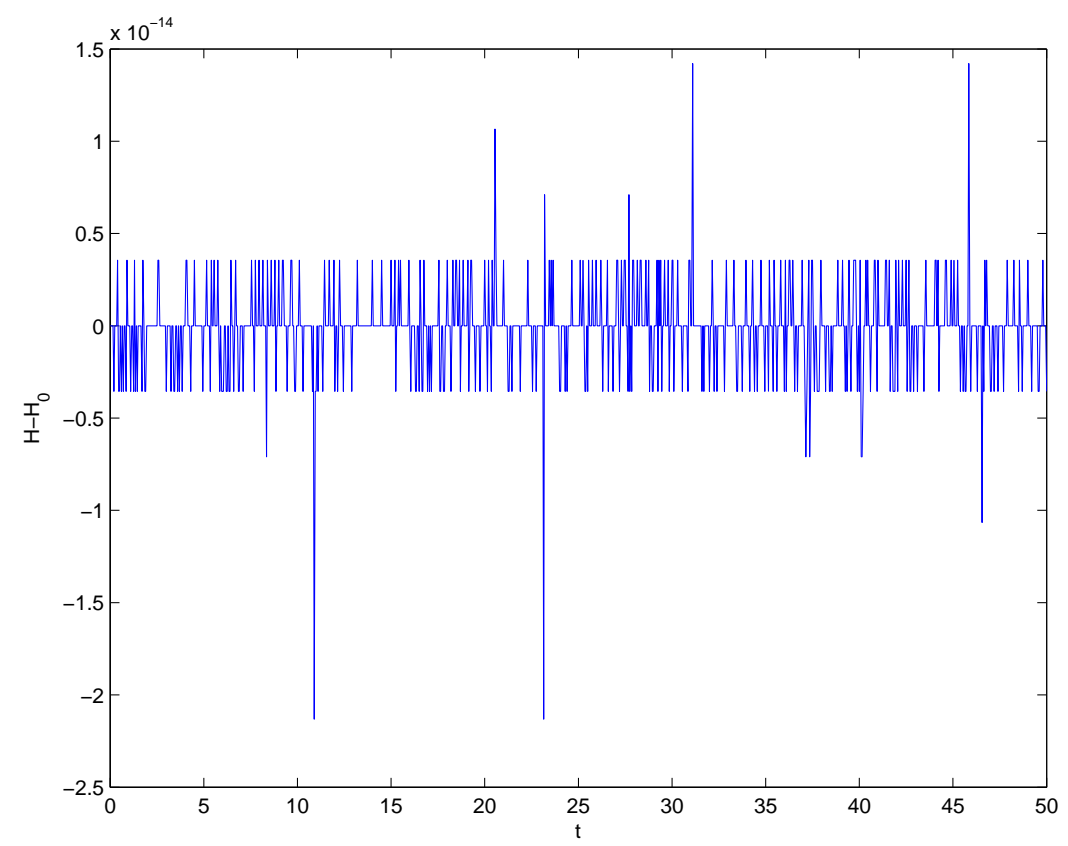

Fig. 5.4 Fourth-order $\operatorname{HBVM}(4,2)$ method, $h=0.05$, problem 5.2]. 
result improves if we increase $k$ : the plot in Figure 5.8 has been obtained by using the $\operatorname{HBVM}(6,2)$, from which one realizes that a practical preservation of the Hamiltonian is reached. Finally, the data listed in Table 5.3 confirm the fourth-order convergence of the latter method.

\section{Conclusions}

In this paper a new class of numerical methods, able to preserve polynomial Hamiltonians, has been studied in details. From the analysis, it turns out that such methods can be regarded as a generalization of collocation Runge-Kutta Lobatto IIIA methods. Nevertheless, the fact of being characterized by a matrix pencil, perfectly fits the framework of block BVMs, so that we have named them Hamiltonian BVMs (HBVMs). A number of numerical tests prove their effectiveness in preserving the Hamiltonian function when evaluated along the numerical solution, as well as confirm the predicted order of convergence. Possible different choices of the abscissae, as well as the actual efficient implementation of the methods, will be the subject of future investigations.

\section{Acknowledgements}

We wish to thank prof. Mario Trigiante for providing us with problem (5.3). We are grateful to Ernst Hairer, for his comments on the proof of Corollary 2 We also thank the referees, for their useful comments and suggestions.

\section{Appendix: some properties of shifted Legendre polynomials}

A number of useful properties of shifted Legendre polynomials are here summarized: for their proof see any book on special functions (e.g., [1]).

P1. Generalized Rodrigues formula: for all $n=0,1, \ldots, P_{n}(x)$ has degree $n$ and can be defined as

$$
P_{n}(x)=\frac{1}{n !} \frac{d^{n}}{d x^{n}}\left[\left(x^{2}-x\right)^{n}\right]
$$

P2. Lobatto quadrature: the Lobatto abscissae $\left\{c_{i}\right\}$ [1.4], of the formula of degree $2 s$, are the zeros of the polynomial

$$
\left(x^{2}-x\right) P_{s}^{\prime}(x),
$$

where $P_{s}^{\prime}(x)$ denotes the derivative of $P_{s}(x)$. The corresponding weights (1.7) are given by:

$$
b_{i}=\frac{1}{s(s+1)\left(P_{s}\left(c_{i}\right)\right)^{2}}, \quad i=0,1, \ldots, s,
$$

which are, therefore, all positive. 


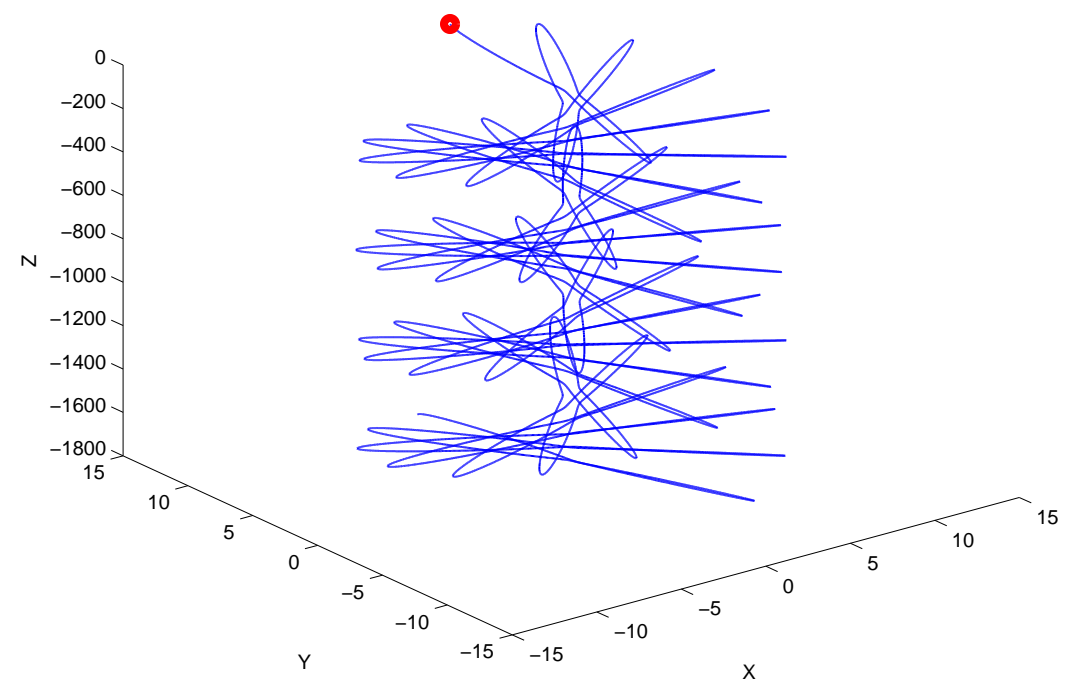

Fig. 5.5 Phase-space plot of the solution of problem 5.3 for $0 \leq t \leq 10^{3}$ (the circle denotes the starting point of the trajectory).

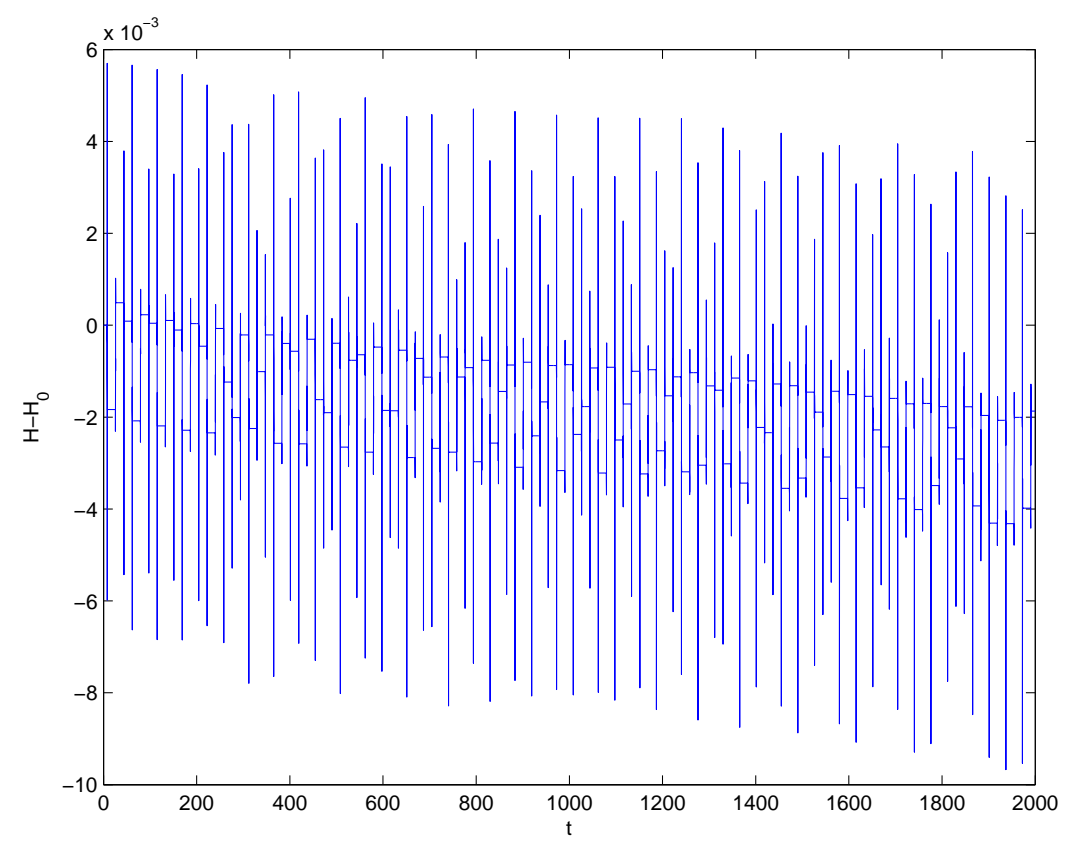

Fig. 5.6 Fourth-order Lobatto IIIA method, $h=0.1$, problem 5.3 . 


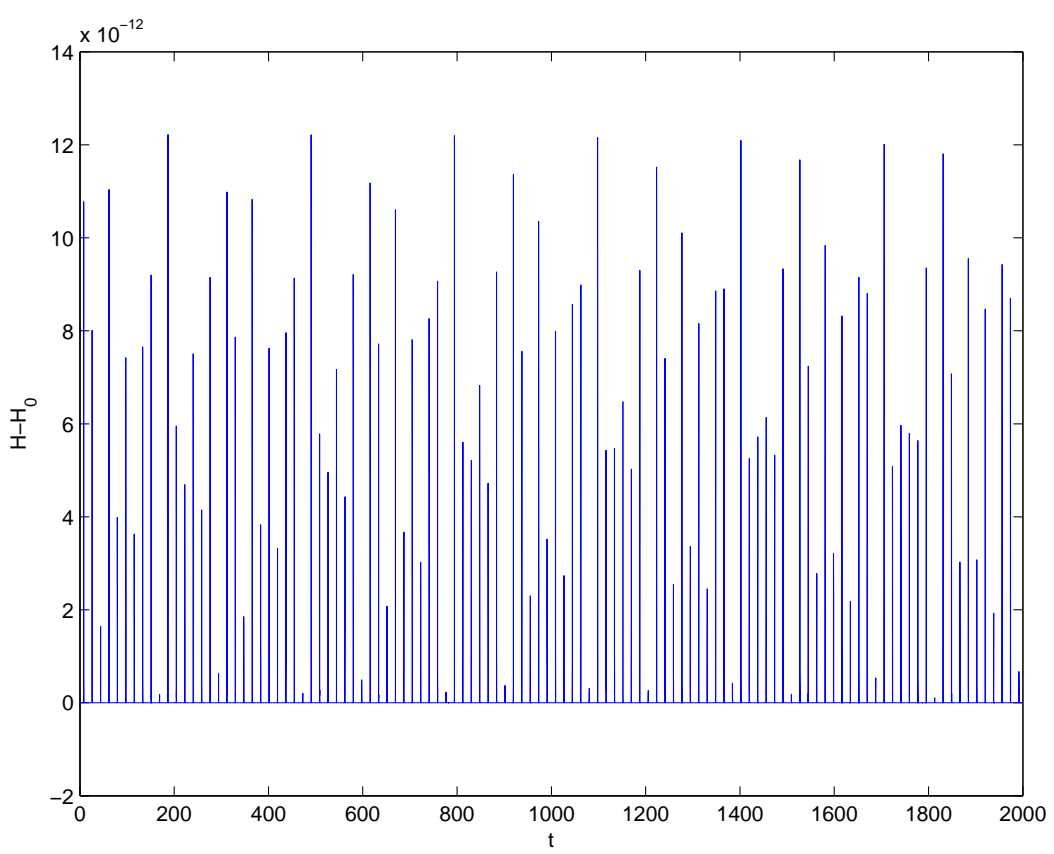

Fig. 5.7 Fourth-order $\operatorname{HBVM}(4,2)$ method, $h=0.1$, problem 5.3.

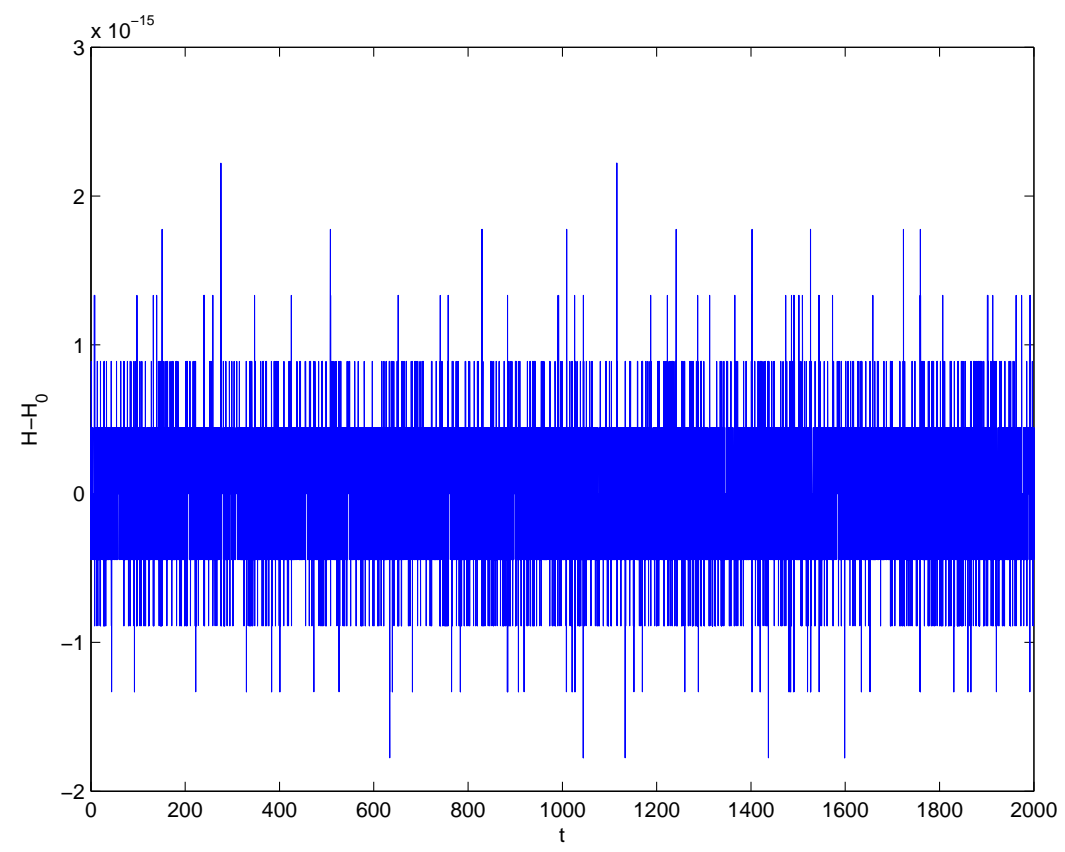

Fig. 5.8 Fourth-order $\operatorname{HBVM}(6,2)$ method, $h=0.1$, problem 5.3. 
P3. Orthogonality:

$$
\int_{0}^{1} P_{n}(x) P_{m}(x) d x=\frac{1}{2 n+1} \delta_{n m}, \quad n=0,1, \ldots,
$$

where, as usual, $\delta_{n m}$ denotes the Kronecker delta.

P4. Recurrence formula: by setting hereafter $P_{-1}(x) \equiv 0$ and $P_{0}(x) \equiv 1$,

$$
(n+1) P_{n+1}(x)=(2 n+1)(2 x-1) P_{n}(x)-n P_{n-1}(x), \quad n=0,1, \ldots .
$$

P5. Explicit formula:

$$
P_{n}(x)=(-1)^{n} \sum_{i=0}^{n}\left(\begin{array}{c}
n \\
i
\end{array}\right)\left(\begin{array}{c}
n+i \\
i
\end{array}\right)(-x)^{i}, \quad n=0,1, \ldots
$$

P6. Symmetry:

$$
P_{n}(1-x)=(-1)^{n} P_{n}(x), \quad n=0,1, \ldots
$$

P7. Symmetry at the end-points:

$$
P_{n}(0)=(-1)^{n}, \quad P_{n}(1)=1, \quad n=0,1, \ldots
$$

P8. Derivatives:

$$
2(2 n+1) P_{n}(x)=\frac{d}{d x}\left[P_{n+1}(x)-P_{n-1}(x)\right], \quad n=0,1, \ldots
$$

P9. Integrals:

$$
\begin{aligned}
2 \int_{0}^{x} P_{0}(t) d t & =2 x=P_{1}(x)+P_{0}(x), \\
2(2 n+1) \int_{0}^{x} P_{n}(t) d t & =P_{n+1}(x)-P_{n-1}(x), \quad n=1,2, \ldots
\end{aligned}
$$

P10. Shifted Legendre differential equations. The shifted Legendre polynomials satisfy the second order differential equation:

$$
\frac{d}{d x}\left[\left(x^{2}-x\right) P_{n}^{\prime}(x)\right]+n(n+1) P_{n}(x)=0, \quad n=0,1, \ldots
$$

P11. From P2 and P10, it follows that, if (1.4) are the Lobatto abscissae of the formula of order $2 s$ (i.e., exact for polynomials of degree $2 s-1$ ), then

$$
\int_{0}^{c_{i}} P_{s}(x) d x=0, \quad i=0,1, \ldots, s .
$$

P12. A few examples:

$$
\begin{aligned}
& P_{0}(x) \equiv 1 \\
& P_{1}(x)=2 x-1 \\
& P_{2}(x)=6 x^{2}-6 x+1 \\
& P_{3}(x)=20 x^{3}-30 x^{2}+12 x-1,
\end{aligned}
$$




\section{References}

1. M. Abramovitz, I.A. Stegun. Handbook of Mathematical Functions. Dover, 1965.

2. L. Brugnano, F. Iavernaro, T. Susca. Hamiltonian BVMs (HBVMs): implementation details and applications. "Proceedings of ICNAAM 2009", AIP Conf. Proc. 1168 (2009) 723-726.

3. L. Brugnano, D. Trigiante. Solving Differential Problems by Multistep Initial and Boundary Value Methods, Gordon and Breach Science Publ., Amsterdam, 1998.

4. L. Brugnano, D. Trigiante. Energy drift in the numerical integration of Hamiltonian problems. J. Numer. Anal. Ind. Appl. Math. 4 (2009) (to appear).

5. E. Celledoni, R. I. McLachlan, D. . McLaren, B. Owren, G. R. W. Quispel, W. M. Wright. Energy preserving Runge-Kutta methods. M2AN 43 (2009) 645-649.

6. P. Chartier, E. Faou, A. Murua. An algebraic approach to invariant preserving integrators: The case of quadratic and Hamiltonian invariants. Numer. Math. 103 (2006) 575-590.

7. E. Faou, E. Hairer, T.-L.Pham. Energy conservation with non-symplectic methods: examples and counter-examples. BIT Numerical Mathematics 44 (2004) 699-709.

8. E. Hairer, C. Lubich, G. Wanner. Geometric Numerical Integration, $2^{\text {nd }}$ ed., Springer, Berlin, 2006.

9. E. Hairer, G. Wanner. Solving Ordinary Differential Equations II, $2^{\text {nd }}$ ed., Springer, Berlin, 1996.

10. F. Iavernaro, B. Pace. $s$-Stage Trapezoidal Methods for the Conservation of Hamiltonian Functions of Polynomial Type. AIP Conf. Proc. 936 (2007) 603-606.

11. F. Iavernaro, B. Pace. Conservative Block-Boundary Value Methods for the Solution of Polynomial Hamiltonian Systems. AIP Conf. Proc. 1048 (2008) 888-891.

12. F. Iavernaro, D. Trigiante. Discrete conservative vector fields induced by the trapezoidal method. $J$. Numer. Anal. Ind. Appl. Math. 1 (2006) 113-130.

13. F. Iavernaro, D. Trigiante. High-order symmetric schemes for the energy conservation of polynomial Hamiltonian problems. J. Numer. Anal. Ind. Appl. Math. 4,1-2 (2009) 87-111.

14. B. Leimkuhler, S. Reich. Simulating Hamiltonian Dynamics. Cambridge Univ. Press, 2004.

15. R.I. McLachlan, G.R.W. Quispel, N. Robidoux. Geometric integration using discrete gradient. Phil. Trans. R. Soc. Lond. A 357 (1999) 1021-1045.

16. G. R. W. Quispel, D. I. McLaren. A new class of energy-preserving numerical integration methods. $J$. Phys. A: Math. Theor. 41 (2008) 045206 (7pp). 